\title{
Subspace Based Blind Channel Estimation for OFDM by Exploiting Virtual Carrier
}

\author{
Chengyang Li and Sumit Roy \\ Dept. of Electrical Engineering, University of Washington, Seattle, WA 98195-2500
}

\begin{abstract}
In this paper, a new subspace based blind channel estimation method is proposed for OFDM systems over a time-dispersive channel. The proposed algorithm exploits the presence of virtual carriers and eliminates the cyclic pre$f i x$; thereby leading to higher channel utilization than most previously reported cyclic prefix based estimators. A sufficient condition on the channel identifiability is developed. Comparison with two other cyclic prefix based subspace methods is presented via computer simulations to support the effectiveness of the proposed method.
\end{abstract}

\section{INTRODUCTION}

Orthogonal Frequency-Division Multiplexing [1], [2] (OFDM) is a promising candidate for high data rate wireless communications for its many advantages - notably, its high spectral efficiency, robustness to frequency selective fading, as well as the feasibility of low-cost transceiver implementations. It has become the European standard for digital audio broadcasting (DAB, target rates $\mathbf{1 . 7}$ $\mathrm{Mbps}$ ). It is also being developed internationally for use in high-speed wireless LANs (e.g. IEEE 802.11a[3], target rates 6-54 Mbps) and wireless-local-loop applications (1-10 Mbps).

While coherent detection is desirable for high data rate communications, it requires reliable estimation of the channel. In the practical OFDM systems operating over a dispersive channel, a Cyclic Prefix (CP) longer than the channel duration is usually inserted in the transmitted sequence. It is well known that this converts the linear (time-domain) convolution between the channel and the input into cyclic convolution or equivalently a (complex) multiplicative factor on each sub channel in the frequency domain. Thus, a training sequence is sent to estimate the frequency domain gains on each sub channel that are used to compensate for the channel scaling; see [4], [2] for overview and [5], [6] for recent results on these lines. The length of the $\mathrm{CP}$ is chosen for the maximum anticipated multipath spread; for the IEEE 802.11a standard, this is $25 \%$ of an OFDM symbol duration, indicating a significant loss in utilization. Additionally, due to the time-varying nature of the channel in wireless applications, the training sequence needs to be transmitted periodically, causing further loss of channel throughput.

This work was supported in part by AFOSR Grant F49620-1-0472 and NSF/ITR CCR-0086032
The above naturally suggests efforts centered around blind channel estimation methods that avoid the need for training and/or even the CP. Recently, the CP has been found to possess useful information that facilitates blind or semi-blind channel estimation [7], [8], [9]. Specifically, Heath and Giannakis[7] proposed a spectrum fitting blind method based on the cyclostationarity property of the time-varying correlation of the received data samples due to the CP insertion at the transmitter; however, this approach suffers from slow convergence of the estimator. Most recently, Cai and Akansu [8] developed a noise subspace method by utilizing the structure of the filtering matrix introduced by the CP insertion that achieves faster convergence for smaller data records while [9] derived a semi-blind approach to track the channel variations using the CP samples (prior to the CP removal) at the receiver. Other than the $\mathrm{CP}$, there exists another resource Virtual Carriers (VC) ${ }^{1}$, as in the IEEE 802.11a standard that specifies that 12 out of the total of 64 sub carriers are left unmodulated. While they were intended to help limiting the transmit spectrum, the VCs can be exploited for the purposes of channcl cqualization or frequency offset estimation as has already been shown by [10], [11]. A method that exploits the presence of VCs and the finite alphabet property of the input data was presented in [12] to develop a Maximum Likelihood joint blind estimator of the channel and the data symbols.

In this paper, we propose a new subspace based channel estimator for an OFDM system without the CP, motivated by the observation that the presence of the $\mathrm{VC}$ enables the extraction of the noise subspace from the observation space. The proposed algorithm stands out from other reported subspace based methods ( [7], [8]) and is attractive for its potential to achieve higher channel utilization because of the CP elimination.

A baseband signal model for the OFDM system is introduced in Section II. The subspace channel estimator is developed in Section III. A sufficient condition on the channel identifiability is provided there as well. Computer simulations are presented in Section IV to demonstrate

\footnotetext{
${ }^{1}$ In the practical FFT-based OFDM systems, a shaping filter is required to limit the transmitted signal spectrum to the desired band. In order to ease filter implementation, some sub carriers in the rolloff region, i.e. the band edge, of the filter are left unmodulated; these are referred to as virtual carriers [4].
} 
the effectiveness of the proposed algorithm with comparison to the two contemporary subspace methods [7], [8]. Finally, paper concludes in Section V with some remarks.

The notation used in this paper follows usual convention - vectors are denoted by symbols in boldface, $(\cdot)^{*}$, $(\cdot)^{T},(\cdot)^{H}$ are complex conjugate, transpose and conjugate transpose of $(\cdot)$, respectively. $\operatorname{rank}(\cdot)$ is the rank of $(\cdot) \cdot \operatorname{ran}(\cdot),\|\cdot\|$, and $(\cdot)^{+}$give respectively the range, Frobenius norm, and Pseudo-inverse of matrix argument.

\section{Signal Formulation}

Consider an OFDM system (see Fig. 1) without $C P$ insertion with $Q$ sub carriers, of which only $P$ are modulated by user's data symbols; i.e. the remaining $Q-P$ unmodulatcd carricrs constitutc virtual carricrs. $\Lambda$ ssume the sub carriers numbered $p_{0}$ to $p_{0}+P-1$ are used for data, where $p_{0}$ is the index of the first data carrier. Let the $k$ th block of the 'frequency domain' information symbols be

$$
\mathbf{s}(k)=\left[s_{0}(k), s_{1}(k), \ldots, s_{P-1}(k)\right]^{T} .
$$

Note for information symbol duration of $T$, the corresponding OFDM symbol duration $T_{s}=Q T$. After multicarrier modulation implemented by IFFT, the 'time domain' output signal vector is given by

$$
\mathbf{x}(k)=\left[x_{Q-1}(k), \ldots, x_{1}(k), x_{0}(k)\right]^{T}=\mathbf{W}_{P} \mathbf{s}(k),
$$

where $\mathbf{W}_{P}$ is the 'row-flipped' version of the $Q \times P$ partial IDFT matrix

$\mathbf{W}_{P}=\frac{1}{\sqrt{Q}}\left[\begin{array}{ccc}W_{Q}^{-p_{0}(Q-1)} & \ldots & W_{Q}^{-\left(P+p_{0}-1\right)(Q-1)} \\ \vdots & & \vdots \\ W_{Q}^{-2 p_{0}} & \ldots & W_{Q}^{-2\left(P+p_{0}-1\right)} \\ W_{Q}^{-p_{0}} & \ldots & W_{Q}^{-\left(P+p_{0}-1\right)} \\ 1 & \ldots & 1\end{array}\right]$

with $W_{Q}=e^{-j 2 \pi / Q}$.

Each element of $\mathbf{x}(k)$ is then pulse shaped by $g_{t r}(t)$ to generate the continuous time signal sent on the channel

$$
x(t)=\sum_{k=-\infty}^{+\infty} \sum_{p=0}^{Q-1} x_{p}(k) g_{i r}\left(t-p T-k T_{s}\right)
$$

Substituting $T_{s}=Q T$ in (3) leads to

$$
x(t)=\sum_{k=-\infty}^{+\infty} \sum_{p=0}^{Q-1} x_{p}(k) g_{t r}(t-(p+k Q) T)
$$

Thus denoting $q=p+k Q$, we identify $k=\left\lfloor\frac{q}{Q}\right\rfloor(\lfloor x\rfloor$ is the largest integer contained in $x$ ) and $p=q$ modulo $Q$. Then the transmitted signal $x(t)$ can be rewritten as

$$
x(t)=\sum_{q=-\infty}^{+\infty} x_{q} g_{t r}(t-q T)
$$

During the transmission, the signal $x(t)$ passes through a dispersive channel with impulse response $c(t)$, is contaminated by AWGN noise $n(t)$, and finally is input into a front-end receive filter $g_{r x}(t)$.

Defining the composite channel filter $h(t)=g_{t r}(t) * c(t) *$ $g_{r x}(t)$ and the filtered noise $v(t)=n(t) * g_{r x}(t)$ where $*$ denotes linear convolution, the received signal $r(t)$ is therefore

$$
r(t)=\sum_{q=-\infty}^{+\infty} x_{q} h(t-q T)+v(t)
$$

Assume the composite channel $h(t)$ to have finite support $[0, L T]$ no longer than the OFDM symbol duration $T_{s}$; this implies that any ISI is only restricted to the past neighboring symbol as is generally true for OFDM. A synchronized rate $1 / T$ sampler after $r(t)$ yields

$$
r(i)=r\left(t_{0}+i T\right)=\sum_{l=0}^{L} x_{i-l} h\left(t_{0}+l T\right)+v(i)
$$

where $v(i)=v\left(t_{0}+i T\right)$.

Let $h(l)=h\left(t_{0}+l T\right)$ and

$$
\mathbf{h}=[h(0), h(1), \ldots, h(L)]^{T}
$$

Define an $M Q-L \times M Q$ Toeplitz matrix $\mathcal{H}$ constructed from $\mathbf{h}$

$$
\mathcal{H}=\operatorname{Toeplitz}\left([\mathbf{h}^{T} \underbrace{0 \ldots 0}_{(M Q-L-1)} 0^{\prime} s\right.
$$

Consider an observation interval over $M$ OFDM symbols from $\left(t_{0}+((k-M+1) Q+L) T\right)$ to $\left(t_{0}+((k+1) Q-1) T\right)$. The resulting received signal vector $(M Q-L \times 1)$ is

$$
\begin{aligned}
\mathbf{r}_{M}(k) & =[r((k+1) Q-1), \ldots, r((k-M+1) Q+L)]^{T} \\
& =\mathcal{H}\left[\begin{array}{c}
\mathbf{x}(k) \\
\mathbf{x}(k-1) \\
\vdots \\
\mathbf{x}(k-M+1)
\end{array}\right]+\underbrace{\left[\begin{array}{c}
v((k+1) Q-1) \\
\vdots \\
v((k-M+1) Q+L)
\end{array}\right]}_{\mathbf{n}(k)} \\
& =\mathcal{H} \cdot \underbrace{\left(\mathbf{I}_{M} \otimes \mathbf{W}_{P}\right)}_{\tilde{\mathbf{W}}} \cdot \underbrace{\left[\begin{array}{c}
\mathbf{s}(k) \\
\mathbf{s}(k-1) \\
\vdots \\
\mathbf{s}(k-M+1)
\end{array}\right]}_{\mathbf{S}(k)}+\mathbf{n}(k) \\
& =\underbrace{\mathcal{H} \tilde{\mathbf{W}}}_{\mathcal{A}} \mathbf{S}(k)+\mathbf{n}(k) .
\end{aligned}
$$

where $\mathbf{I}_{M}$ is an $M \times M$ identity matrix and $\otimes$ denotes kronicker product. 


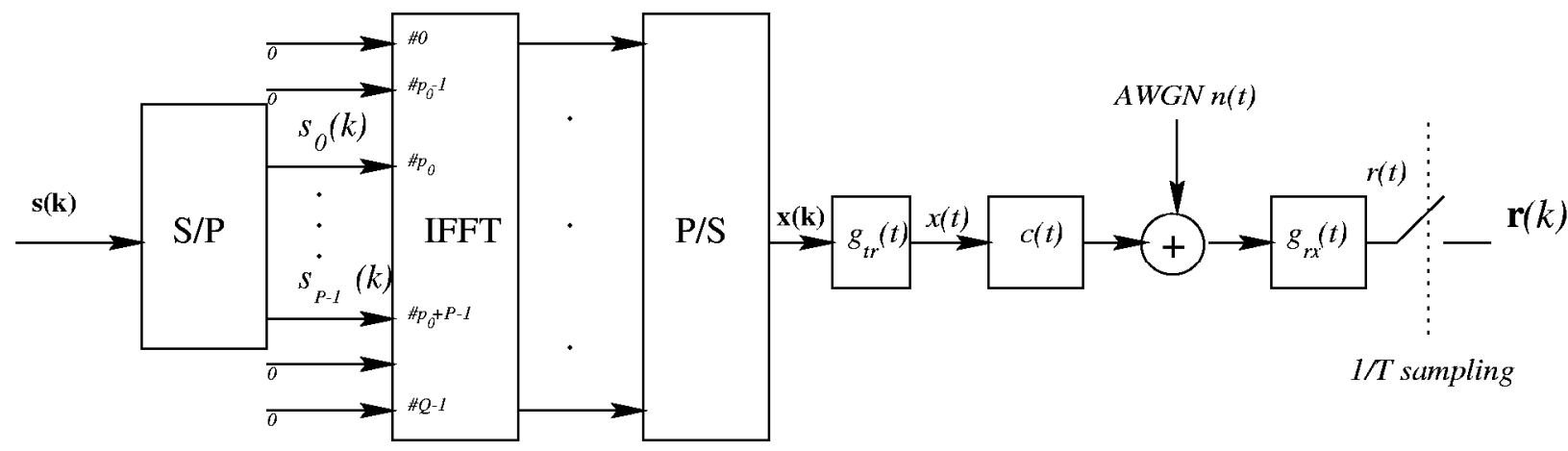

Fig. 1. Baseband OFDM system (without CP) model

Remarks: For the signal model (10) where the equivalent filtering matrix $\mathcal{A}$ dimension is $M Q-L \times M P$, a necessary condition for the subspace method is that $M(Q-P)>L$. Due to the presence of the VCs $Q>P$, the necessary condition can always be satisfied by choosing an appropriate $M$. This condition is assumed to hold throughout the paper.

\section{Subspace Based Channel estimation}

We next state sufficient conditions (Theorems 1 and 2) for the proposed subspace based channel estimator without proof because of space constraint.

\section{A. Sufficient Conditions for Identifiability}

From $(10), \mathcal{A}=\mathcal{H} \tilde{\mathbf{W}}(M Q-L \times M P)$ where $\mathcal{H}$ is an $M Q-L \times M Q$ Toeplitz matrix and $\tilde{\mathbf{W}}=\mathbf{I}_{M} \otimes \mathbf{W}_{P}$ is an $M Q \times M P$ matrix. A sufficient condition for the filtering matrix $\mathcal{A}$ to have full column rank is given in Theorem 1 .

Theorem 1: $\mathcal{A}$ has full column rank, i.e., $\operatorname{rank}(\mathcal{A})=$ $M P$, if $(Q-L) \geq P$ and the frequency response of $\mathbf{h}$ has no nulls at any of the data sub carrier frequencies.

In the derivation hereafter, we assume the above sufficient condition is satisfied. Also the user's transmitted information symbols $s_{i}(k)$ 's are i.i.d. sequences with zero mean and known variance $\sigma_{s}^{2}\left(\sigma_{s}^{2}=1\right.$ without loss of generality). In addition, Nyquist pulse shaping is employed so that each element of $\mathbf{n}(k)$ in (10) is AWGN with zero mean and variance $\sigma_{n}^{2}$. After collecting $N_{b}$ signal vectors, we have

$$
\begin{aligned}
\mathbf{Y} & =\left[\mathbf{r}_{M}(\mathbf{1}), \ldots, \mathbf{r}_{M}\left(N_{b}\right)\right] \\
& =\mathcal{A} \underbrace{\left[\mathbf{S}(1), \ldots, \mathbf{S}\left(N_{b}\right)\right]}_{\mathbf{S}}+\mathbf{N} \\
& =\mathbf{X}+\mathbf{N}
\end{aligned}
$$

Applying the Singular Value Decomposition (SVD) on the unpurturbed received signal matrix $\mathbf{X}$ yields

$$
\mathbf{X}=\mathcal{A} \mathbf{S}=\left[\begin{array}{ll}
\mathbf{U}_{s} & \mathbf{U}_{n}
\end{array}\right]\left[\begin{array}{ll}
\boldsymbol{\Sigma}_{s} & \\
& \mathbf{0}
\end{array}\right]\left[\begin{array}{l}
\mathbf{V}_{s}^{H} \\
\mathbf{V}_{n}^{H}
\end{array}\right]
$$

where $\left[\mathbf{U}_{s}, \mathbf{U}_{n}\right]$ is an $M Q-L \times M Q-L$ unitary matrix. The $M P$ columns of $\mathbf{U}_{s}$ span the signal subspace, while $M(Q-P)-L$ column vectors of $\mathbf{U}_{n}$ span the subspace (often known as the noise subspace as in practice the SVD is applied on the noise purturbed signal matrix $\mathbf{Y}$ ) orthogonal to the signal subspace. $\boldsymbol{\Sigma}_{s}=\operatorname{diag}\left(\lambda_{1}, \lambda_{2}, \ldots, \lambda_{M P}\right)$ is a diagonal matrix consisting of $M P$ significant singular values corresponding to the signal subspace. The orthogonality property between the signal subspace and the noise subspace asserts

$$
\mathbf{U}_{n}(i)^{H} \mathcal{A}=0(i=1, \ldots, M(Q-P)-L)
$$

where $\mathbf{U}_{n}(i)$ is the $i$-th column of $\mathbf{U}_{n}$.

From (13), the channel vector $\mathbf{h}$ can be uniquely identified up to a complex scaling factor under conditions given in Theorem 2 below.

Theorem 2: Let $\mathbf{h}^{\prime}$ and $\mathbf{h}$ denote two distinct solutions to (13); $\mathcal{A}^{\prime}$ be the corresponding matrix constructed using $\mathbf{h}^{\prime}$ as with $\mathcal{A}$ in (10). For $M \geq 2$, if $\operatorname{ran}\left(\mathcal{A}^{\prime}\right)=\operatorname{ran}(\mathcal{A})$, then $\mathbf{h}^{\prime}=\alpha \mathbf{h}$ where $\alpha$ is a complex scalar.

Remarks:

1) The presence of the VCs is necessary for the filtering matrix $\mathcal{A}$ to be full column rank. Unique identification of the channel is guaranteed by the specific structure of $\tilde{\mathbf{W}}$. 2) The condition $Q-L \geq P$ requires the number of the VCs be greater than the channel memory. This is satisfied in typical OFDM application scenarios.

3) The condition that the channel's frequency response does not have a null at any of the data carriers appears restrictive. However, in such a case, any information on the sub channel is lost anyway; the method proposed in [8] fails in this situation as well. Solution to this loss of information is to use receive diversity or coding across sub channels, for example.

\section{B. Blind Channel Estimator}

Let

$$
\mathbf{U}_{n}(i)=\left[u_{i}(0), u_{i}(1), \ldots, u_{i}(M Q-L-1)\right]^{T}
$$


Exploiting the special structure of $\mathcal{H}$ yields

$$
\mathbf{U}_{n}(i)^{T} \mathcal{H}=\overline{\mathbf{h}}^{T} \mathcal{U}_{i}
$$

where the $L+1 \times M Q$ dimension matrix $\mathcal{U}_{i}$ is generated from the vector $\mathbf{U}_{n}(i)$

$$
\mathcal{U}_{i}=\operatorname{Toeplitz}\left([\mathbf{U}_{n}^{T}(i) \underbrace{0 \ldots 0}_{L 0^{\prime} s}]\right)
$$

When only an estimate of the noise subspace $\hat{\mathbf{U}}_{n}$ is available in practice, (13) suggests the channel estimator

$$
\hat{\mathbf{h}}=\arg \min _{\|\mathbf{h}\|=1} \sum_{i=1}^{M(Q-P)-L}\left\|\hat{\mathbf{U}}_{n}(i)^{H} \mathcal{A}\right\|^{2}
$$

But from (15)

$$
\begin{aligned}
\left\|\mathbf{U}_{n}(i)^{H} \mathcal{A}\right\|^{2} & =\mathbf{U}_{n}(i)^{H} \mathcal{H} \mathbf{\mathbf { W }} \tilde{\mathbf{W}}^{H} \mathcal{H}^{H} \mathbf{U}_{n}(i) \\
& =\mathbf{h}^{T} \underbrace{\left(\mathcal{U}_{i}\right)^{*} \tilde{\mathbf{W}}}_{\mathbf{G}_{i}} \underbrace{\tilde{\mathbf{W}}^{H} \mathcal{U}_{i}^{T}}_{\mathbf{G}_{i}^{H}} \mathbf{h}^{*}
\end{aligned}
$$

Thus, by defining $\tilde{\mathbf{h}}=(\mathbf{h})^{*}$, and

$$
\mathcal{G}=\left[\mathbf{G}_{1}, \ldots, \mathbf{G}_{M(Q-P)-L}\right],
$$

the channel information $\tilde{\mathbf{h}}$ is determined by

$$
\hat{\tilde{\mathbf{h}}}=\arg \min _{\|\tilde{\mathbf{h}}\|=1} \tilde{\mathbf{h}}^{H} \hat{\mathcal{G}} \hat{\mathcal{G}}^{H} \tilde{\mathbf{h}}
$$

where $\hat{\mathcal{G}}$ is the estimate of $\mathcal{G}$. It is well known that $\tilde{\mathbf{h}}$ (or equivalently $\mathbf{h}$ ) is the eigenvector corresponding to the smallest eigenvalue of the matrix $\hat{\mathcal{G}} \hat{\mathcal{G}}^{H}$.

\section{SIMULATION RESULTS}

Monte Carlo simulations were conducted to assess the effectiveness of the proposed blind estimator with comparison to other two reported subspace channel estimation methods for OFDM [7], [8]. The number of sub carriers $Q=15$. Note that our proposed method eliminates the CP, while the methods of [7], [8] used as comparison baselines require the $\mathrm{CP}$, whose length is set to 4 as in [7], [8].

To evaluate the estimation error, the normalized Root Mean Squared Error(RMSE)

$$
\operatorname{RMSE}=\frac{1}{\|\mathbf{h}\|} \sqrt{\frac{1}{N_{m}(L+1)} \sum_{p=1}^{N_{m}}\left\|\hat{\mathbf{h}}_{p}-\mathbf{h}\right\|^{2}}
$$

is used, where the subscript $p$ refers to the $p$-th simulation run and $N_{m}$ denotes the number of runs. Information sequence $s_{i}(k)$ 's are BPSK modulated. Input

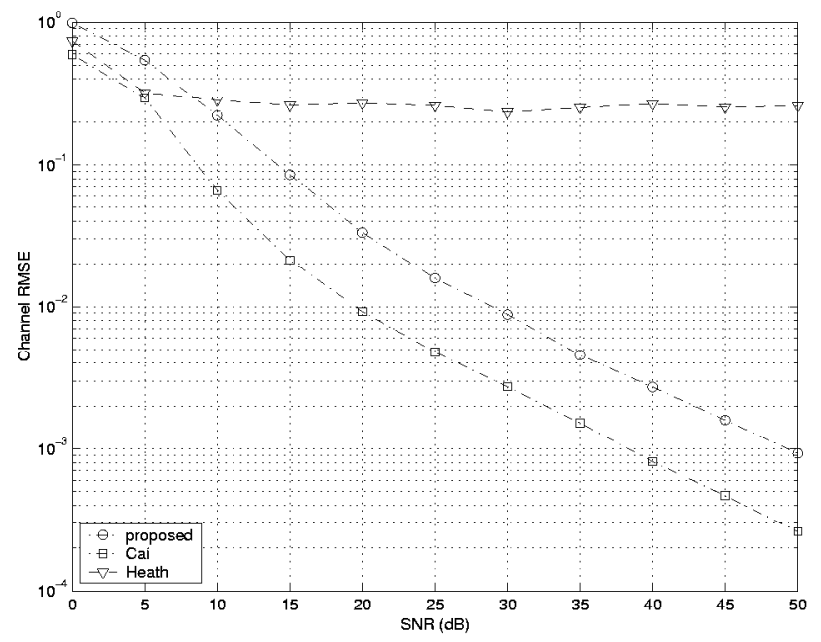

Fig. 2. Channel error versus SNR $\left(N_{b}=120\right)$

SNR is defined as SNR $\stackrel{\text { def }}{=} 10 \log _{10} \frac{\sigma_{s}^{2}}{\sigma_{n}^{2}}$. The same tworay multipath channel $h(t)=e^{-j 2 \pi(0.15)} r_{c}(t-T / 2, \beta)+$ $0.8 e^{-j 2 \pi(0.6)} r_{c}(t-1.2 T, \beta)$ as [7], [8] is used, where $r_{c}(t)$ is raised cosine with roll-off factor $\beta=0.35$. The channel is sampled at $t=0, T, \ldots, 4 T$; hence $L=4$. For the proposed method, the number of the VCs is set to 4 (i.e., $P=11$ ) unless otherwise noted. Smoothing factor $M$ is set to 2 for our method and [8]. Results shown are the average over 300 runs for all three methods, i.e., $N_{m}=300$.

Fig. 2 shows the estimator error as a function of the input SNR's using $N_{b}=120$. And Fig. 3 illustrates the error as a function of the number of data blocks $N_{b}$ for $\mathrm{SNR}=15 \mathrm{~dB}$. As expected, the estimator error of all three methods decreases with increasing input SNR and the data record length $N_{b}$. Also notable is that both our approach and the method in [8] (Cai) perform much better than that of [7] (Heath), reflecting the fast convergence property of the noise subspace estimator for small data record. Although there is a performance gap between the proposed method and [8], the proposed estimator achieves low error $(<0.1)$ when SNR $=15 d B$ with only 120 OFDM blocks, suggesting it a possible candidate for wideband communication scenarios where the channel is time-invariant for only a few OFDM symbols. Moreover, the proposed method avoids the CP and therefore leads to higher throughput than [8].

Finally, the dependence of the proposed estimator error on the number and the location of the available $\mathrm{VCs}$ is investigated in Fig. 4, using SNR $=40 d B$ and $N_{b}=300$. The number of the VCs is varied from 4 to 7 (correspondingly, the number of the data carriers $P$ is changing from 11 down to 8). Results corresponding to three different locations - on the low frequency side of the spectrum only $\left(p_{0}=Q-P\right.$, marked as 'low'), on the high frequency side of the spectrum only ( $p_{0}=0$, marked as 'high'), and on 


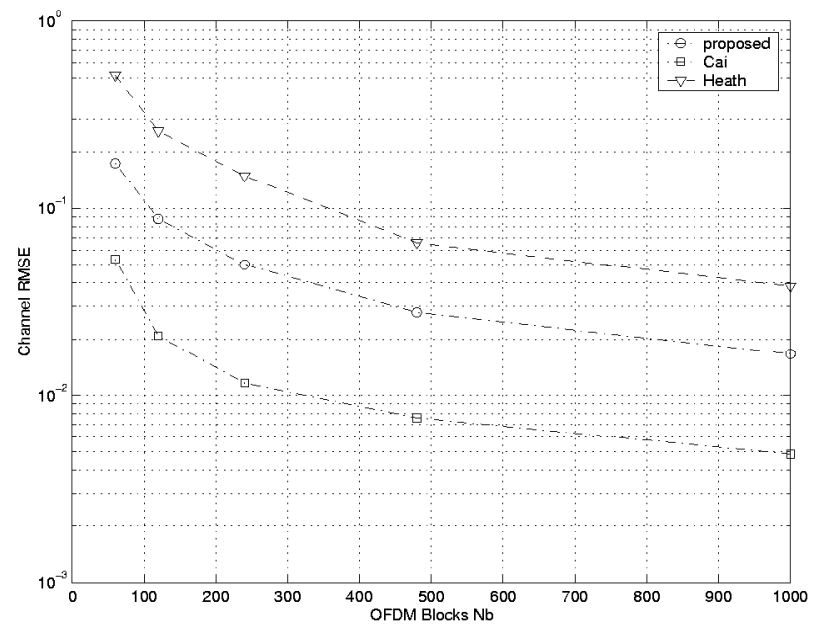

Fig. 3. Channel error versus number of data blocks $N_{b}$ (SNR $=$ $15 d B)$

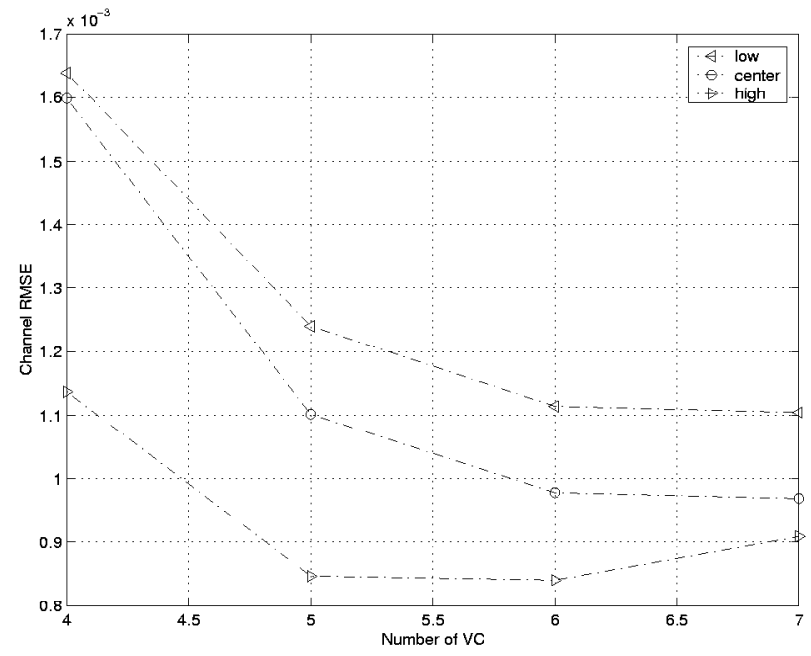

Fig. 4. RMSE vs. number of VC

both sides of the spectrum $\left(p_{0}=\left\lfloor\frac{Q-P}{2}\right\rfloor\right.$, marked as 'center') - are plotted together. As is seen, different location of the VCs affects the estimator performance. And more VCs in the system improves the estimate accuracy; this is reasonable since larger number of the VCs means a larger dimension of the noise subspace $(M(Q-P)-L)$, yielding more constraints on the channel vector (as in (13)).

\section{Conclusion}

Redundancy in the practical OFDM system - training symbols, CP, VC and pilot tones - offers many opportunities to estimate the channel. In this paper, we presented a subspace based blind channel estimator for a noCP OFDM system by taking advantage of the presence of the VCs. A sufficient condition on the identifiability was established as well. The algorithm is attractive for its potential to increase the system's channel utilization due to the elimination of the CP. Comparison of the proposed method with other two reported subspace channel estimation methods by computer simulation illustrates good performance of the proposed method with regard to both estimate accuracy and speed of convergence.

\section{REFERENCES}

[1] J.A.C. Bingham, "Multicarrier modulations for data transmission: An idea whose time has come", IEEE Commun. Mag., vol. 28, no.5, pp. 5-14, May 1990.

[2] W.Y. Zou and Y. Wu, "COFDM: An overview", IEEE Trans. Broadcasting, vol. 41, pp. 1-8, Mar. 1995.

[3] IEEE 802.11a, "Wireless LAN Medium Access Control (MAC) and Physical Layer (PHY) specifications: High speed physical layer in the 5 GHZ band", IEEE Standard, 1999.

[4] H.Sari, G.Karam, and I.Jeanclaude, "Transmission techniques for digital terrestrial TV broadcasting", IEEE Commun. Mag., vol. 33, no.2, pp. 100-109, Feb. 1995 .

[5] O. Edfors, M.Sandell, J. van de Beek, S.Kate, and P.O.Borjesson, "OFDM channel estimation by singular value decomposition", IEEE Trans. on Commun., vol. 46, no.7, pp.931-939, July 1998 .

[6] Y. Li, L.J.Cimini Jr., and N.R.Sollenberger, "Robust channel estimation for OFDM systems with rapid dispersive fading channels", IEEE Trans. on Commun., vol. 46, no. 7, pp.902915 , July 1998.

[7] R.W.Heath and G.B.Giannakis, "Exploiting Input Cyclostationarity for blind channel identification in OFDM systems", IEEE Trans. Sig. Proc., vol 47,no.3, pp. 848-856, Mar. 1999.

[8] Xiaodong Cai and Ali N. Akansu, "A subspace method for blind channel identification in OFDM systems", in Proc. of ICC2000, pp.929-933, 2000.

[9] Xiaowen Wang and K.J.Ray Liu, "Adaptive Channel Estimation Using Cyclic Prefix in Multicarrier Modulation System", IEEE Commun. letters, vol. 3, no. 10, pp.291-293, Oct. 1999.

[10] Marc de Courville, P.Madec and J.Palicot, "Blind Dqualization of OFDM System based on the Minimization of a Quadratic Criterion", in Proc. ICC 96, 1996, pp1318-1322.

[11] U. Tureli and H. Liu, "A high-efficiency carrier estimator for OFDM communications", IEEE Commun. Letters, vol. 2, no.4, pp.104-106, Apr. 1998.

[12] Yu Song, S.Roy and L.A. Akers "Joint blind estimation of channel and data symbols in OFDM", VTC 2000, Spring, 2000. 